\title{
Aggressive characteristics of lattice model
}

\author{
Lv Feng ${ }^{1, a}$ \\ ${ }^{1}$ Department of Basic Education, Zhejiang Agricultural Business College, \\ Shaoxing 312088, China \\ alvfzhi@163.com
}

Keywords: Aggressive; Lattice model; Critical point

Abstract. In this paper, a new lattice model of traffic flow with the consideration of the aggressive-driving behavior is investigated by Xiaoqin-Li et al. The model is studied by using linear and nonlinear analysis method. A thermodynamic theory is transformed to describe the phase transition and critical phenomenon. The time-dependent Ginzburg-Landau (TDGL) equation is derived of near the critical point.

\section{Introduction}

Recently, many studies attraction to the density wave investigation for the lattice models[1-4]. The jamming transitions have the properties very similar to the conventional phase transition. The TDGL equation can describe the non-equilibrium phase transition. Now, few lattice models could deduce the TDGL equation. It is necessary to construct the traffic flow models by which one can derive the TDGL equation since the thermodynamic theory of jamming transition can be formulated by the TDGL equation. The lattice models can not describe the complex phenomena resulted by the driver's aggressive characteristics that they didn't include this factor.

\section{Car-following model and linear stability analysis}

In real traffic, most of aggressive drivers adjusts them speed by estimating the impending traffic information of the next-nearest car. In view of the above reason, a new lattice model of traffic flow with the consideration of the aggressive-driving behavior(for short, DAE) is presented by Xiaoqin-Li et al[5]. The motion equation is given as follows:

$$
\begin{gathered}
\partial_{t} \rho_{j}+\rho_{c}\left(p_{j} v_{j}-p_{j-1} v_{j-1}\right)=0 \\
\rho_{j}(t+\tau) v_{j}(t+\tau)=\rho_{c}\left[(1-p) V\left(\rho_{j+1}(t)\right)+p V\left(\rho_{j+2}(t+\tau)\right)\right]
\end{gathered}
$$

The optimal velocity function $V\left(\rho_{j}\right)$ is adopted as below [6]:

$$
V\left(\rho_{j}\right)=\frac{V_{\max }}{2}\left[\tanh \left(\frac{1}{\rho_{j}}-\frac{1}{\rho_{c}}\right)+\tanh \left(\frac{1}{\rho_{c}}\right)\right]
$$


For convenience of analysis, Eq.(1) and Eq.(2) can be rewritten:

$$
\begin{gathered}
\partial_{t} \rho_{j}(t+\tau)+\rho_{c}^{2}\left\{\left[(1-p) V\left(\rho_{j+1}\right)+p V\left(\rho_{j+2}\right)\right]-\left[(1-p) V\left(\rho_{j}\right)+p V\left(\rho_{j+1}\right)\right]\right\} \\
-k\left(\partial_{t} \rho_{j+2}-\partial_{t} \rho_{j+1}\right)=0
\end{gathered}
$$

where $k=-p \tau \rho_{c}^{2} V^{\prime}\left(\rho_{j+2}(t)\right)$.

Therefore, we can derive the TDGL equation from Eq.(4), which could describe traffic jams in terms of a kink density wave. Then, linear stability analysis can be conducted for DAE.

Suppose $y_{j}(t)$ to be small deviations from the steady-state solution $\rho_{c}$ :

$$
\rho_{j}(t)=\rho_{c}+y_{j}(t)
$$

By inserting Eq.(5) into Eq.(4), and expanding $y_{j}=A \exp (i k j+z t), \quad z=z_{1}(i k)+z_{2}(i k)^{2}+\mathrm{L}$, then the first- and second-order terms of $i k$ are:

$$
\begin{aligned}
& z_{1}=-\rho_{c}{ }^{2} V^{\prime}\left(\rho_{c}\right), \\
& z_{2}=-\left[\frac{1+2 p}{2}+\rho_{c}{ }^{2} V^{\prime}\left(\rho_{c}\right) \tau+k\right] \rho_{c}{ }^{2} V^{\prime}\left(\rho_{c}\right) .
\end{aligned}
$$

For small disturbances with long wavelengths, the uniform traffic flow is stable in the condition that

$$
\tau<-\frac{1+2 p+2 k}{2 \rho_{c}^{2} V^{\prime}\left(\rho_{c}\right)}
$$

\section{TDGL equation}

The slow scales for space variable $\mathrm{j}$ and time variable $\mathrm{t}$ are introduced, and the slow variables $\mathrm{X}$ and $\mathrm{T}$ are set as follows[5]:

$$
X=\varepsilon(j+b t), \quad T=\varepsilon^{3} t, \quad 0<\varepsilon<<1 .
$$

The density $\rho_{j}(t)$ is defined as $\rho_{j}(t)=\rho_{c}+\varepsilon R(X, T)$

By inserting Eqs. (8)-(9) into Eq. (4), and expanding to the fifth-order of $\varepsilon$. We obtain the expression:

$\varepsilon^{2} m_{1} \partial_{X} R+\varepsilon^{3} m_{2} \partial_{X}^{2} R+\varepsilon^{4}\left[\partial_{T} R+m_{3} \partial_{X}^{3} R+m_{4} \partial_{X} R^{3}\right]+\varepsilon^{5}\left[m_{5} \partial_{X} \partial_{T} R+m_{6} \partial_{X}^{4} R+m_{7} \partial_{X}^{2} R^{3}\right]=0$

(10)where

$m_{1}=b+\rho_{c}{ }^{2} V^{\prime}, \quad m_{2}=b^{2} \tau-k b+\frac{1+2 p}{2} \rho_{c}{ }^{2} V^{\prime}, \quad m_{3}=\frac{b^{3} \tau^{2}}{2} V^{\prime}-\frac{3 b k}{2}+\frac{1+b p}{6} \rho_{c}{ }^{2} V^{\prime}$,

$m_{4}=\frac{V^{\prime \prime \prime}}{6} \rho_{c}^{2}, \quad m_{5}=2 b \tau-k, \quad m_{6}=\frac{b^{4} \tau^{3}}{6}-\frac{7 k b}{6}+\frac{1+14 p}{24} \rho_{c}{ }^{2} V^{\prime}, \quad m_{7}=\frac{V^{\prime \prime \prime}(1+2 p)}{12} \rho_{c}{ }^{2}$.

Now, we study the traffic flow near critical point $\tau=\left(1+\varepsilon^{2}\right) \tau_{c}$. By taking $b=-\rho_{c}{ }^{2} V^{\prime}$, the senond- and third-order terms of $\varepsilon$ from Eq. (10) is eliminated. Thus leads to the simplified equation as following 
$\varepsilon^{4} \partial_{T} R=\varepsilon^{4} n_{1} \partial_{X}^{3} R-\varepsilon^{4} n_{2} \partial_{X} R^{3}-\varepsilon^{3} n_{3} \partial_{X}^{2} R-\varepsilon^{5} n_{4} \partial_{X}^{4} R+\varepsilon^{5} n_{5} \partial_{X}^{2} R^{3}$

Where

$$
\begin{aligned}
& n_{1}=\frac{12 \tau_{c}-21-18 p-8 \tau_{c}{ }^{2}}{8}, \\
& n_{2}=\frac{V^{\prime \prime \prime}}{6} \rho_{c}{ }^{2}, \\
& n_{3}=\tau-\tau_{c}, \\
& n_{4}=\frac{2 \tau_{c}{ }^{3}-12 \tau_{c}{ }^{2}+4 \tau_{c}-25-20 p}{12}, \\
& n_{5}=\frac{V^{\prime \prime \prime}(1-2 p)}{12} \rho_{c}{ }^{2} .
\end{aligned}
$$

In order to derive the TDGL equation, we should define

$$
\frac{n_{4}}{n_{1}}=\frac{n_{5}}{n_{2}}=q
$$

By transforming $X$ and $T$ to variables $x=\varepsilon^{-1} X$ and $t=\varepsilon^{-3} T$, and taking $S(x, t)=\varepsilon R(X, T)$, Eq. (11) is rewritten as follows:

$\partial_{t} S=n_{1} \partial_{x}^{3} S-n_{2} \partial_{x} S^{3}-n_{3} V \partial_{x}^{2} S-q n_{1} \partial_{x}^{4} S+q n_{2} \partial_{x}^{2} S^{3}$.

By adding term $\frac{n_{3}}{q} \partial_{x}^{2} S$ to both the left- and right-hand sides and performing Galilean transformation $t_{1}=t$ and $x_{1}=x-\frac{n_{3}}{q} t$ for Eq. (12), we get

$$
\partial_{t_{1}} S=\left(\partial_{x_{1}}-q \partial_{x_{1}}^{2}\right)\left\{n_{1} \partial_{x_{1}}^{2} S+\frac{n_{3}}{q} S-n_{2} S^{3}\right\}
$$

We define the thermodynamic potentials:

$$
\phi(S) \equiv-\frac{n_{3}}{2 q} S^{2}+\frac{n_{2}}{4} S^{4}
$$

By rewritten Eq. (13) with Eq. (14), the TDGL equation is derived

$$
\begin{gathered}
\partial_{t_{1}} S=-\left(\partial_{x_{1}}-q \partial_{x_{1}}^{2}\right) \frac{\delta \Phi(S)}{\delta S} \\
\Phi(S) \equiv \int d x_{1}\left[q n_{1}\left(\partial_{x_{1}} S\right)^{2}+\phi(S)\right]
\end{gathered}
$$

where $\delta \Phi(S) / \delta S$ indicates the function derivative. The TDGL Eq. (16) has two steady-state solutions except for a trivial solution $S=0$ : one is the uniform solution:

$$
S\left(x_{1}, t_{1}\right)= \pm\left[\frac{m_{3}}{m_{2}}\right]^{\frac{1}{2}}
$$


and the other is the kink solution

$$
S\left(x_{1}, t_{1}\right)= \pm\left[\frac{m_{3}}{m_{2}}\right]^{\frac{1}{2}} \times \tanh \left\{\left[\frac{m_{3}}{m_{1}}\right]^{\frac{1}{2}} \times\left(x_{1}-x_{0}\right)\right\}
$$

\section{Summary}

We have obtained the neutral stability line and the critical point by using the liner stability theory for the DAE. The TDGL equation has been derived to describe traffic behavior near the critical point by applying the reductive perturbation method.

\section{Acknowledgements}

This work was financially supported by the Zhejiang Agricultural Business College, China (Grant No. KY201605).

\section{References}

[1] D. Helbing, B. Tilch: Generalized force model of traffic dynamics. Phys. Rev. E, 58, 133(1998). [2] T. Nagatani: TDGL and MKDV equations for jamming transiton in the lattice models of traffic. Phys. A, 264, 581 (1999).

[3] T.Q. Tang, H.J. Huang and H.Y. Shang: A new macro model for traffic flow with the consideration of the driver's forecast effect. Phys. Lett. A, 374, 1668-1672(2010).

[4] H.X. Ge, R.J. Cheng and S.M. Lo: Time-dependent Ginzburg-Landau equation for lattice hydrodynamic model describing pedestrian flow. Chin. Phys. B, 22(7) 070507 (2013).

[5] X.Q.Li, K.L. Fang and G.H. Peng: A new lattice model of traffic flow with the consideration of the drivers' aggressive characteristics. Phys. A, 468 315-321 (2016).

[6] M. Bando, K. Hasebe, A. Nakayama, A. Shibata and Y. Sugiyama: Dynamical model of traffic congestion and numerical simulation. Phys.Rev. E, 51, 1035(1995). 